\title{
Six-Dimensional Energy-Switching Potential Energy Surface for HeHCN
}

\author{
Wazir-ul H. Ansari ${ }^{\dagger}$ and António J. C. Varandas* \\ Departamento de Química, Universidade de Coimbra 3004-535 Coimbra, Portugal
}

Received: May 14, 2002; In Final Form: August 2, 2002

\begin{abstract}
A single-valued six-dimensional (6D) potential energy surface is determined for $\mathrm{HeHCN}$ by using the energy switching (ES) method, which utilizes a global double many-body expansion (DMBE) as well as a Legendre polynomial expansion for different energy regimes. The ES potential has a linear van der Waals well of 29.5 $\mathrm{cm}^{-1}$ which is about $4 \mathrm{~cm}^{-1}$ lower than that reported by Drucker et al. [J. Phys. Chem. 1995, 99, 2646] but agrees with one obtained by Atkins and Hutson [ J. Chem. Phys. 1996, 105, 440]. The effect of stretch of CN as well as $\mathrm{CH}$ bonds has also been investigated. With stretch, both the DMBE and ES schemes yield slightly deeper wells and steeper repulsion walls in the high energy region. Microwave transitions calculated at 15.9 and $31.3 \mathrm{GHz}$ agree well with experiment and may be assigned to $J=1 \leftarrow 0$ and $J=2 \leftarrow 1$, respectively. This frequency is found to decrease with stretch. Finally, this ES potential energy surface has been compared with that of ArHCN reported by one of us [Chem. Phys. Lett. 1998, 297, 458]. Here, the question of transferability of the parameter which scales the Hatree-Fock energy in the rare gas-X $(\mathrm{X}=\mathrm{H}, \mathrm{C}$, and $\mathrm{N})$ interaction from $\mathrm{ArHCN}$ to $\mathrm{HeHCN}$ has also been investigated. The transferability seems to work as it nicely brings out the position of the potential well and the rovibrational levels. The microwave transition, from the ground state $(j=0, L=0, J=0)$ to state $(1,0,1)$, found at $98.61 \mathrm{GHz}$ in $\mathrm{HeHCN}$ can be compared with one found at about $162.48 \mathrm{GHz}$ in $\mathrm{ArHCN}$, in close agreement with experiment.
\end{abstract}

\section{Introduction}

During the last two decades, there has been increased interest in the study of intermolecular interactions of rare gas $(\mathrm{Rg})-$ HCN van der Waals complexes. ${ }^{1-15}$ Monomers and dimers were investigated both experimentally and theoretically. ${ }^{3,4,9,16-23}$ An ab initio CEPA-I and MP4 potential energy surface for ArHCN was reported by Clary et al. ${ }^{2}$ and Tao et al., ${ }^{22}$ respectively. A host of workers ${ }^{17,18,21,24,25}$ experimentally investigated this van der Waals complex, although arriving at no general consensus on the form of its potential energy surface. ${ }^{22}$ Gutowsky et al., ${ }^{3}$ while investigating experimentally the microwave rotational transitions of $\mathrm{Ne}-\mathrm{HCN}$ dimer, have also presented a molecularmechanics-for-clusters (MMC) potential energy surface for Rg$\mathrm{HCN}$ dimers with $\mathrm{He}, \mathrm{Ne}, \mathrm{Ar}$, and $\mathrm{Kr}$ as rare gas giving stabilities of $21,37,85$, and $108 \mathrm{~cm}^{-1}$, respectively. Their results also show a quasilinear equilibrium geometry for both $\mathrm{He}-$ $\mathrm{HCN}$ and $\mathrm{Ne}-\mathrm{HCN}$ dimers.

The first ab initio potential energy surface for the title van der Waals molecule, good enough to reproduce experimental data on high-resolution ground-state spectroscopy, has been reported a few years ago by Drucker et al. ${ }^{4}$ They calculated rovibrational energies and wave functions at the MP4 level using a large basis set containing bond functions. Their function has a well of $25 \mathrm{~cm}^{-1}$ at the center of mass separation $R=4.27 \AA$, with a global minimum occurring at the collinear configuration $\mathrm{He} \cdot \cdot \mathrm{HCN}$, and the minimum energy rising monotonically with large angular-radial coupling, as the $\mathrm{HCN}$ orientation angle $\theta$ increases from 0 to $2 \pi$. Drucker et al. ${ }^{4}$ have also reported highresolution spectroscopy of the bound states of HeHCN. Ground-

* To whom correspondence should be addressed. E-mail: varandas@ qtvs1.qui.uc.pt.

$\dagger$ On leave from Department of Physics, LN Mithila University, Darbhanga-846004, India. state $J=1 \leftarrow 0$ and $J=2 \leftarrow 1$ transitions were measured at 15893.6108 and $31325.2443 \mathrm{MHz}$, respectively. Their rovibrational energies calculated from an intermolecular Legendretype potential energy surface agree to within $10 \%$ with observed values of $\mathrm{mm}$ wave/microwave transition energies.

This work was followed by Atkins and Hutson, ${ }^{5}$ who obtained two potential energy surfaces (1E8 and 2E8) for the title system by least-squares fitting to data from high-resolution microwave and millimeter-wave spectroscopy of Drucker et al. ${ }^{4}$ The global minimum of $1 \mathrm{E} 8$ is at a linear configuration $\mathrm{He} \cdot \cdots \mathrm{HCN}$ with a significantly deeper well of depth $29.47 \mathrm{~cm}^{-1}$, whereas $2 \mathrm{E} 8$ has a slightly nonlinear well of $29.36 \mathrm{~cm}^{-1}$. Using the potential energy surfaces, they predict new spectral lines which yet require confirmation.

More recently, Toczylowski et al. ${ }^{15}$ reported two-dimensional potential energy surface for $\mathrm{Rg}-\mathrm{HCN}$, with $\mathrm{Rg}=\mathrm{He}, \mathrm{Ne}, \mathrm{Ar}$, and $\mathrm{Kr}$, using the $\operatorname{CCSD}(\mathrm{T})$ approach and the augmented correlation consistent polarized basis sets with an additional set of bond functions, with a linear well of $29.9 \mathrm{~cm}^{-1}$ at $R=4.22$ $\AA$ and a local bent minimum of depth $22.07 \mathrm{~cm}^{-1}$ at $R=3.59$ $\AA$. They also predict bound-state spectral lines that are yet to be confirmed.

The Taylor series or polynomial expanded potential energy functions may accurately reproduce the available vibrationalrotational data, because they are designed to be valid in the vicinity of the potential well to which the fitted data belongs. However, they may not be good enough for our understanding of the dynamics of van der Waals molecular complexes if one looks at the bond-breaking/formation of the chemically stable molecule. For this, one requires a fully dimensional potential energy surface which describes any product channel (including the van der Waals one). The HeHCN potential energy functions of Atkins and Hutson ${ }^{5}$ assume the form of a single-channel atom-pseudo-diatom Legendre expansion, with the radial co- 
efficients being in turn written as a Legendre analysis. They also include some empirical elements, which make them reproduce accurately the available rovibrational data. For this work, we have chosen their form $1 \mathrm{E} 8$, because this is somewhat simpler and both are expected to lead to very similar results. Of course, we could have equally employed the form $2 \mathrm{E} 8$, but this was unavailable to us. The reader should consult the original paper for further details.

A global double many-body expansion (DMBE) $)^{26-28}$ potential energy surface for ArHCN was presented by one of us ${ }^{7}$ for investigating the dynamics of recombination/dissociation of $\mathrm{HCN}$, with the observation that the precise form of the attractive well is relatively unimportant in scattering dynamics and that it is rather the inner repulsive wall that could play a very important role in the dynamics of the system. So, in view of these and similar observations made by Bruehl et al. ${ }^{29}$ and Hippler et al., ${ }^{30}$ the global DMBE potential energy surface, which employs the realistic extended Hartree-Fock approximate correlation energy ${ }^{26,31,32}$ (EHFACE2U) model, should provide a reliable representation of the potential energy both in the repulsive wall and long-range regions.

The DMBE potential energy surface for ArHCN was found good enough for a detailed dynamics study of the dissociation reaction $\mathrm{Ar}+\mathrm{HCN} \rightarrow \mathrm{Ar}+\mathrm{H}+\mathrm{CN}^{7}{ }^{7}$ The calculated thermal rate constant was found to be in good agreement with experiment. As the DMBE surface was not found as much good for the bound states of the system, a global ES potential function was then considered by the authors. ${ }^{7}$ This global ES potential energy surface ${ }^{8}$ has been used to investigate the spectra of van der Waals modes for the ArHCN molecule. Such a surface combines the global DMBE form with a Legendre-type expansion (or a form similar to one used by Tao et al. ${ }^{22}$ or other related form, if one so wishes) for the system. This then appears to have a promise of reproducing both the kinetics and rovibrational spectroscopy.

The 6D DMBE surface, or the ES one which entails it, can also be used to investigate an important aspect of molecular structure, associated with bond stretching, as it may be related to the features of the inner repulsive wall of the potential energy surface. The importance of proper representation of the attractive part of the potential surface cannot be overemphasized for the bound states.

While investigating $\mathrm{Rg}-\mathrm{HCN}$ van der Waals systems, we revisited our work on $\mathrm{Ar}-\mathrm{HCN}^{8}$ and found that a slightly different repulsive function could represent its potential better (see below). Moreover, many-body expanded functions apart from describing correct dissociative behavior have the advantage of providing a data bank $\mathrm{k}^{33}$ of potentials of all fragments needed to build up a polyatomic potential and can be used as a prototype potential.

In view of the above, therefore, we report, in this work, a global 6D energy-switching potential energy surface for $\mathrm{HeHCN}$, along with our results of its application to study the effect of bond stretching on one hand and the microwave transitions of $\mathrm{HeHCN}$ on the other, using a slightly different repulsive function than the one we reported for ArHCN and some empirical elements as used by Atkins and Hutson, ${ }^{5}$ deferring report of our results on ArHCN. The structure of this paper is as follows. In section 2, we review the methodology. Sections 3 and 4 contain the discussion of results and the concluding remarks, respectively.

\section{Methodology}

2.1. DMBE Potential Energy Surface. As the ES potential energy surface is constructed from the DMBE and Legendre's expanded functions, we first briefly describe the DMBE methodology, the details of which may be found elsewhere. ${ }^{26-28,34}$ Accordingly, the four-body 6D potential for $\mathrm{HeHCN}$ may be written as a sum of a realistic DMBE potential energy surface for $\mathrm{HCN}$ and relevant two-body potentials involving $\mathrm{He}$ atom, with the three-body and four-body terms involving $\mathrm{He}$ atom ignored, as a first approximation

$$
V(\mathbf{R})=V_{\mathrm{HCN}}\left(R_{\mathrm{CN}}, R_{\mathrm{CH}}, R_{\mathrm{NH}}\right)+\sum_{\beta=\mathrm{C}, \mathrm{H}, \mathrm{N}} V_{\mathrm{He} \beta}\left(R_{\mathrm{He} \beta}\right)
$$

where $\mathbf{R}$ is a collective variable that denotes the six internuclear distances of the system, $V_{\mathrm{HCN}}$ is the three-body $\mathrm{HCN}$ potential, ${ }^{35}$ and the terms under summation sign represent the realistic Hartree-Fock approximate correlation energy (denoted EHFACE2 $\mathrm{U}^{26,31,32}$ ) and may, under DMBE scheme be written for He interactions as follows:

$$
V_{\mathrm{He} \beta}=\lambda_{\mathrm{He} \beta} V_{\mathrm{HF}}(R)+V_{\mathrm{dc}}(R)
$$

where $\lambda_{\mathrm{He} \beta}$ is a scaling parameter fitted to obtain a satisfactory van der Waals potential for $\mathrm{HeHCN}$ and $V_{\mathrm{HF}}(R)$ represents the repulsive interaction of Born-Meyer type and may be given by

$$
V_{\mathrm{HF}}(R)=A \exp \left(-b R-c R^{2}\right)
$$

which may also be written as $V_{\mathrm{HF}}(R)=\exp \left(a-b R-c R^{2}\right)$ for pairs of atoms involving He. $A$ (or $a$ ), $b$, and $c$ are parameters obtained from a least-squares fit to restricted open-shell Hartree-Fock (RHF) ab initio calculations as discussed below. The second term, $V_{\mathrm{dc}}(R)$, of the right-hand side of eq 2 is the dynamical correlation energy, and it has been modeled ${ }^{36}$ semiempirically by the damped dispersion series expansion

$$
V_{\mathrm{dc}}(R)=-\sum_{n=6,8,10} \chi_{n}(R) C_{n} / R^{n}
$$

where $C_{n}$ are the dispersion energy coefficients and $\chi_{n}$ are dispersion damping functions defined as ${ }^{26}$

$$
\chi_{n}(R)=\left[1-\exp \left(-A_{n} x-B_{n} x^{2}\right)\right]^{n}
$$

where $x=R / \rho$ is a reduced coordinate and $\rho$ a scaling parameter given by 26,32

$$
\rho=5.5+1.25 R_{0}
$$

with $R_{0}$ being defined the LeRoy distance ${ }^{37}$ for onset of the undamped $R^{-n}$ expansion. In turn, for two interacting atoms $\mathrm{A}$ and $\mathrm{B}$, one has

$$
R_{0}=2\left(\left\langle r_{\mathrm{A}}{ }^{2}\right\rangle^{1 / 2}+\left\langle{r_{\mathrm{B}}}^{2}\right\rangle^{1 / 2}\right)
$$

with $\left\langle r_{\mathrm{A}}{ }^{2}\right\rangle$ and $\left\langle r_{\mathrm{B}}{ }^{2}\right\rangle$ being the expectation values of the squared radii for the outermost electrons in atoms $\mathrm{A}$ and $\mathrm{B}$, respectively; $A_{n}$ and $B_{n}$ are given in terms of universal parameters $\alpha_{0}, \alpha_{1}$, $\beta_{0}$, and $\beta_{1}$ as $A_{n}=\alpha_{0} n^{-\alpha_{1}}$ and $B_{n}=\beta_{0} \exp \left(-\beta_{1} n\right)$, respectively, where $\alpha_{0}=16.36606, \alpha_{1}=0.70172, \beta_{0}=17.19338$, and $\beta_{1}=$ 0.09574. The values of other parameters are given in Table 1 .

2.2. ES Potential Energy Surface. An accurate global energy-switching strategy for the potential energy surface of polyatomic systems was presented by one of us. ${ }^{38,39}$ Accordingly, the ES potential energy surface for $\mathrm{HeHCN}$ can be written as follows:

$$
V_{\mathrm{ES}}=f(\Delta E) V_{1}(\mathbf{R})+[1-f(\Delta E)] V_{2}\left(\mathbf{R}^{\prime}\right)
$$


TABLE 1: Parameters of DMBE Potential Energy Surface

\begin{tabular}{|c|c|c|c|c|c|c|c|c|}
\hline & \multirow[b]{2}{*}{$\lambda_{\mathrm{He} \beta}$} & \multirow[b]{2}{*}{$A$} & \multirow[b]{2}{*}{$b$} & \multirow[b]{2}{*}{$c$} & \multirow[b]{2}{*}{$R_{0}$} & \multicolumn{3}{|c|}{ dispersion coefficients } \\
\hline & & & & & & $C_{6}$ & $C_{8}$ & $C_{10}$ \\
\hline $\mathrm{HeH}$ & 0.2047 & 0.327043 & 1.36148 & 0.0456541 & 5.6409 & 2.823 & 41.83 & 871.30 \\
\hline $\mathrm{HeC}$ & 6.2907 & 0.622917 & 1.11593 & 0.103813 & 6.1224 & 7.14 & 116.29 & 2481.36 \\
\hline $\mathrm{HeN}$ & 1.3951 & 2.28306 & 1.60163 & 0.0472255 & 5.4688 & 5.90 & 80.80 & 1448.91 \\
\hline
\end{tabular}

where $V_{1}(\mathbf{R})$ is the DMBE potential energy surface $V(\mathbf{R})$ of eq 1 and $V_{2}\left(\mathbf{R}^{\prime}\right)$ is the function $V(R, \theta)$ used by Atkins and Hutson ${ }^{5}$ added to the global HCN DMBE potential energy surface. ${ }^{35}$ Thus, $V_{2}\left(\mathbf{R}^{\prime}\right)$ is a $5 \mathrm{D}$-dimensional potential energy surface as implied by the prime, which causes no problem when obtaining the final potential energy surface in eq 8 , because this is obviously a function valid over the full-dimensional configuration space $\mathbf{R}$. In turn, $\Delta E$ is defined by

$$
\Delta E=E-E_{0}
$$

and gives the displacement from some reference energy (in the energy space defined by $V_{2}$ ), whereas $f(\Delta E)$ is a switching function that approaches 0 for large negative energy displacements $(\Delta E \rightarrow-\infty)$ and +1 for large positive ones $(\Delta E \rightarrow+\infty)$. $V_{\text {ES }}$ changes smoothly from $V_{1}(\mathbf{R})$ to $V_{2}\left(\mathbf{R}^{\prime}\right)$ as the energy varies across $E_{0}$, which is accomplished by the switching function $f(\Delta E)$. This should be system specific, but following previous work, ${ }^{38,39}$ we may write it as follows:

$$
f(\Delta E)=\frac{1}{2}[1+\tanh (\gamma \Delta E)]
$$

With this form of $f$, eq 8 assumes the form

$$
V_{\mathrm{ES}}=f(\Delta E) V_{1}(\mathbf{R})+f(-\Delta E) V_{2}(\mathbf{R})
$$

An optimum value of $\gamma$ was so determined by trial and error for a selected reference energy, from the requirement that $V_{\mathrm{ES}}$ should give a good description of the rovibrational spectra of $\mathrm{HeHCN}$ and not invite spurious topographical features at other regions of configuration space. Note that $V_{\mathrm{ES}}$ would become $5 \mathrm{D}$ only when $\Delta E \rightarrow-\infty$. Of course, although $f(\Delta E)$ is calibrated to attain very small values at specific regions, it never becomes zero because both merged potential energy surfaces are bounded below.

2.3. Bound States and Microwave Transitions. Rovibrational levels of HeHCN were obtained through solutions of the bound-state Schrödinger equation as a set of coupled differential equations, ${ }^{40}$ using a Hamiltonian of the form

$$
H=-\frac{\hbar^{2}}{2 \mu} R^{-1} \frac{\mathrm{d}^{2}}{\mathrm{~d} R^{2}} R+H_{\mathrm{int}}+V(R, \theta)
$$

where $H_{\text {int }}$ is the internal Hamiltonian of the pseudodiatomic (i.e., $\mathrm{HCN}$ ), and $V(R, \theta)$ is the interaction potential in Jacobi coordinates, including the centrifugal interaction. The former is obtained by subtracting the potential of $\mathrm{HCN}$ from $V_{\mathrm{ES}}(\mathbf{R})$, with the internal coordinates of $\mathrm{HCN}$ frozen at the specific values chosen for the calculation. The BOUND program ${ }^{41}$ was used to solve eq 12, using a space-fixed basis set including all $\mathrm{HCN}$ rotor functions up to $j=14$. The rotational constant of HCN and the reduced mass of the complex were 1.478 221834 $\mathrm{cm}^{-1}$ and $3.486027184 m_{\mathrm{u}}$ respectively. The grid for the $R$ coordinate was determined by $R_{\min }=2 \AA$ and $R_{\max }=10 \AA$ with a step size of $0.05 \AA$. The frequencies of microwave

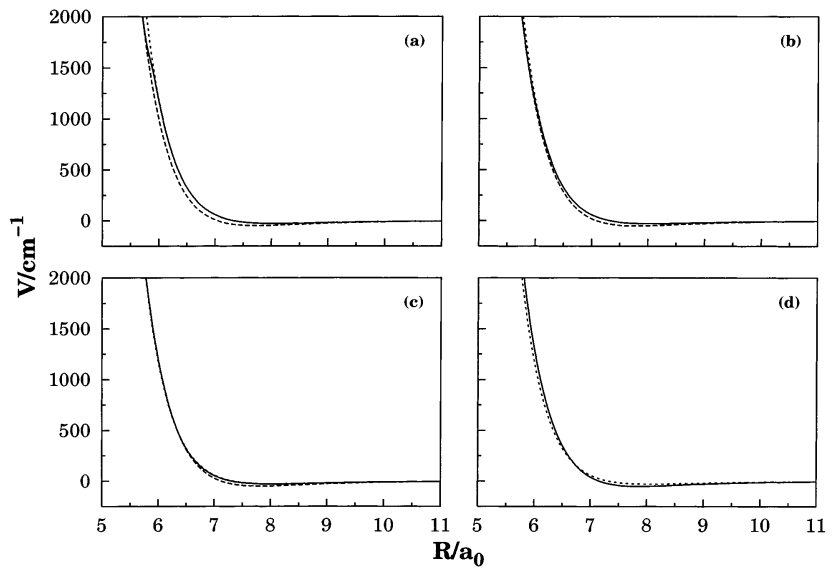

Figure 1. Comparison of plots of $\mathrm{He} \cdots \mathrm{HCN}\left(v_{1}, 0, v_{3}\right)$ potential energy surface by DMBE, ES, and 1E8 methods in vibrational states: (a) $(0$, $0,0)$, (b) $(1,0,2),(\mathrm{c})(2,0,3)$, and (d) $(1,0,5)$.

transitions were then obtained from the differences of corresponding energy levels by using the usual Bohr rules.

\section{Results and Discussion}

To obtain the DMBE potential energy surface, the two-body and three-body interaction energy terms for $\mathrm{HCN}$ were, following spin-spatial Wigner-Witmer rules, treated as in ref 34. For diatomics $\mathrm{HeH}, \mathrm{HeC}$, and $\mathrm{HeN}$, the potentials $V_{\mathrm{HF}}$ were calculated ab initio for ground states ${ }^{2} \Sigma^{+},{ }^{3} \Sigma^{-}$, and ${ }^{4} \Sigma^{-}$, respectively, using the augmented correlation consistent quadruple- $\zeta$ (VQZ) basis sets ${ }^{42-45}$ in a RHF scheme. Calculations were done using the MOLPRO package. ${ }^{46}$ The calculated $\mathrm{ab}$ initio potentials were used to fit the parameters $A$ (or $a$ ), $b$, and $c$ of eq 3 as described above. Table 1 gives the values of these parameters. The asymptotic standard errors are generally within $6 \%$ for bond distances $4<R / a_{0}<10$.

The parameter $\lambda_{\mathrm{He} \beta}$ was determined from consideration of obtaining a correct behavior at dissociation limits as well as a smooth potential well for $\mathrm{He} \cdot \cdot \mathrm{HCN}$ interaction. One way to ensure it could be to minimize the rmsd of this model potential from reference $a b$ initio energies. ${ }^{7}$ We have chosen it in a different way. It is considered that the component $V_{\mathrm{He} \beta}$ of DMBE potential $V(\mathbf{R})$ of eq 1 is mainly coming from the HF energy, and $V_{\mathrm{HF}}(R)$ and its coefficient $\lambda_{\mathrm{He} \beta}$ merely rescales it. So it should have more or less the same value for $\mathrm{He} \cdot \cdots \mathrm{HCN}$ as for $\mathrm{Ar} \cdots \mathrm{HCN}$ interaction, considering the otherwise similarities of Rg-HCN chemistry. Assuming the transferability of such parameters, we have taken them identical to the optimum values found in the ArHCN case. Its value are collected in Table 1.

Such a behavior of DMBE potential, as noted above, is even more important from the standpoint of the ES potential energy surface. In the latter case, the parameters $E_{0}$ and $\gamma$ of eqs 9 and 10 were obtained by trial and error done from the requirement that the ES function should reproduce the lowest microwave transition. This also ensures a smooth rather than a sharp switch from DMBE potential energy surface to a reference $a b$ initio surface as shown in Figure 1a. We have obtained a value of $1709.5 \mathrm{~cm}^{-1}$ for the reference energy $E_{0}$ and $0.003 \mathrm{~cm}^{-1}$ for $\gamma$. 
$1 E 8$

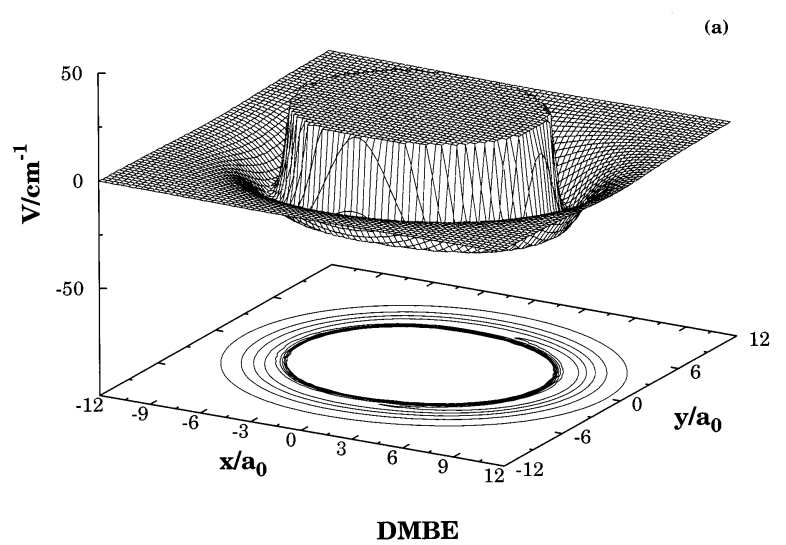

(b)

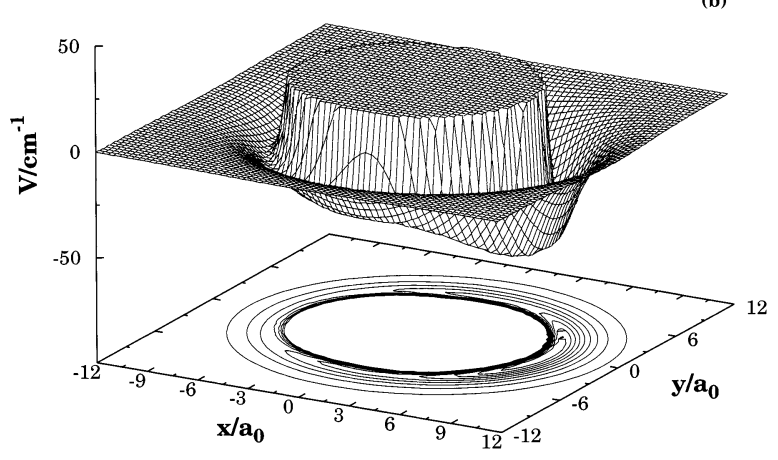

ES

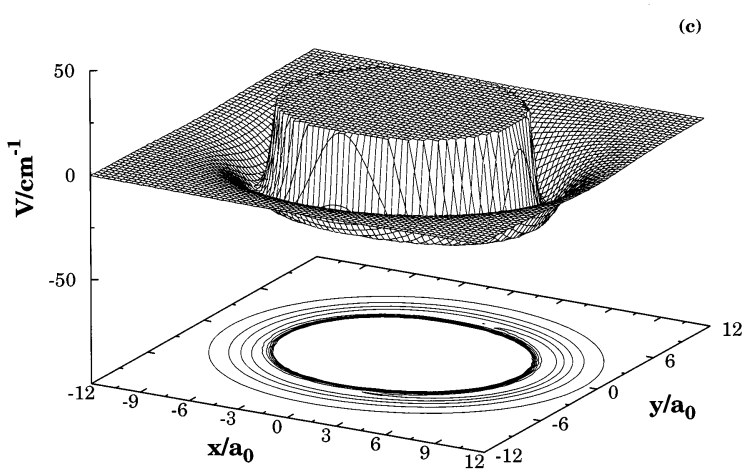

Figure 2. Perspective view/contour plot of $\mathrm{HeHCN}$ potential energy surface for an $\mathrm{He}$ atom moving around an $\mathrm{HCN}$ molecule frozen at its linear equilibrium geometry (a) 1E8, (b) DMBE, and (c) ES.

3.1. Potential Well. A perspective view/contour plot of the $1 \mathrm{E} 8, \mathrm{DMBE}$, and ES potential energy surfaces with $\theta=0$ may be seen in Figure $2 \mathrm{a}-\mathrm{c}$. The plot obtained by the $1 \mathrm{E} 8$ parametrizaton of Atkins and Hutson ${ }^{5}$ has also been included for comparison. All potential energy surfaces exhibit a linear global minimum. The DMBE potential energy surface shows the deepest well $\left(-49.95 \mathrm{~cm}^{-1}\right)$ at $R=4.09 \AA$. The greater depth of the well is expected because of the nonconsideration of some three-body and four-body terms in the expansion. Nevertheless, the DMBE potential energy surface shows a satisfactory asymptotic behavior, which makes it useful for the study of reaction dynamics and kinetics. The ES potential well is $-29.47 \mathrm{~cm}^{-1}$ found at $R=4.27 \AA$ (these values coincide with those of 1E8), in agreement with the well obtained by Atkins and Hutson. ${ }^{5}$

An equal well depth obtained by both the methods, ES and $1 \mathrm{E} 8$, confirms our assumption of transferability of the scaling
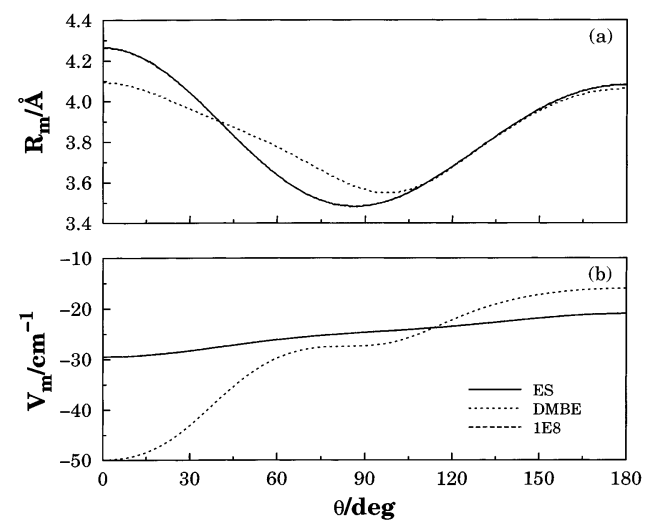

Figure 3. Variation of position and depth of potential well of $\mathrm{HeHCN}$ for $0<\theta<\pi$ : (a) $R_{\mathrm{m}}(\theta)$ and (b) $V_{\mathrm{m}}(\theta)$.

parameter, $\lambda_{\mathrm{x} \beta}$, where $x=\operatorname{Ar}$ or He [cf. eq 2]. It also closely agrees with the well of $-29.90 \mathrm{~cm}^{-1}$ at $R=4.22 \AA$ reported by Toczylowski et al., ${ }^{15}$ using the CCSD (T) scheme. Both Atkins et al., ${ }^{5}$ with $2 \mathrm{E} 8$ parametrization and Toczylowski et al. ${ }^{15}$ report a local minimum also. It is found at somewhat smaller separation of about $3.6 \AA$, with a small nonlinear equilibrium geometry. However, we do not find it, either by DMBE or ES methods, because this is based on 1E8. The ab initio calculations of Drucker et al. ${ }^{4}$ done at MP4 level, upon which Atkins et al. ${ }^{5}$ base their calculations, do not also exhibit such a minimum. Drucker et al., ${ }^{4}$ however, reported a global minimum about $4 \mathrm{~cm}^{-1}$ higher at $R=4.27 \AA$.

With heavier members of the $\mathrm{Rg}-\mathrm{HCN}$ series, for example, the $\mathrm{Ar}-\mathrm{HCN}$, a deeper well with a linear global minimum of about -85.0 and $-173.1 \mathrm{~cm}^{-1}$, respectively, at about $4.63 \AA$ was found by the ES and DMBE methods, ${ }^{7,8}$ respectively. Toczylowski et al. ${ }^{15}$ found it at $147.0 \mathrm{~cm}^{-1}$ at $4.5 \AA$.

The functions $R_{\mathrm{m}}(\theta)$ and $V_{\mathrm{m}}(\theta)$ giving the position of energy minimum and well depth plotted against $\theta$ for $0<\theta<\pi$ are compared in Figure 3. Each of the functions obtained from the ES surface runs coincidently with that obtained from 1E8 surface. The functions obtained from DMBE surface have some differences. Here, the well is deeper and obtained at smaller separation, $R_{\mathrm{m}}(\theta)$, for reasons explained above. The function $R_{\mathrm{m}}(\theta)$ has a minimum at around $\theta=85^{\circ}$ for ES or $1 \mathrm{E} 8$ and around $\theta=100^{\circ}$ for DMBE potential energy surfaces. It is around this minimum that DMBE function $V_{\mathrm{m}}(\theta)$ has a point of inflection. Although $R_{\mathrm{m}}(\theta)$ obtained by the DMBE method runs more or less parallel to the one obtained for the ES or the 1 E8 functions, $V_{\mathrm{m}}(\theta)$ obtained by the DMBE method has an interesting oscillatory dependence on $\theta$. Such an oscillatory behavior is not unexpected as observed by Atkins and Hutson ${ }^{5}$ in the case of the 2E8 parametrization.

3.1.1. Effect of Bond Stretching. With stretch of the $\mathrm{CN}$ or $\mathrm{CH}$ bonds, both the polarizability and short-range repulsion may be expected to increase, lending greater depth to the wells and pushing the repulsive wall away. In fact, the ES potential energy surface shows slightly deeper wells with increasing stretch, with its repulsive wall getting closer to the (unstretched) $1 \mathrm{E} 8$ repusive wall, vide Figure $1 \mathrm{a}-\mathrm{c}$. With still greater stretch, it goes even beyond 1E8, as may be seen in Figure 1d. For this investigation, we have used both the systematic increase of $\mathrm{CN}$ or $\mathrm{CH}$ stretch and the expectation values of the bond lengths for higher vibrational states $\left(v_{1}, v_{2}\right.$, and $\left.v_{3}\right)$, where the subscripts 1 and 3 refer to the normal modes for symmetric and asymmetric stretching of $\mathrm{HCN}$ and 2 refers to its bending normal mode (the bending quantum number $v_{2}$ has been constrained to the 
TABLE 2: Effect of $\mathrm{CN}$ and $\mathrm{CH}$ Stretching on the He $\cdots \mathrm{HCN}$ Potential Well

\begin{tabular}{|c|c|c|c|c|c|c|c|c|c|c|}
\hline \multirow[b]{2}{*}{$\left(v_{1}, v_{2}, v_{3}\right)$} & \multicolumn{2}{|c|}{$\langle r\rangle^{a}$} & \multicolumn{2}{|c|}{$\langle R\rangle$} & \multicolumn{2}{|c|}{$r(\mathrm{H}-\mathrm{C}-\mathrm{N})$} & \multicolumn{2}{|c|}{ ES } & \multicolumn{2}{|c|}{ DMBE } \\
\hline & scaled $^{c}$ & unscaled & scaled $^{c}$ & unscaled & scaled & unscaled & $R_{\mathrm{m}}$ & $\epsilon^{b}$ & $R_{\mathrm{m}}$ & $\epsilon$ \\
\hline 000 & 2.1920 & 2.2020 & 3.1961 & 3.2143 & 4.2080 & 4.2308 & 8.06 & -29.4669 & 7.74 & -49.9460 \\
\hline 100 & 2.2058 & 2.2159 & 3.2061 & 3.2244 & 4.2244 & 4.2474 & 8.06 & -29.4670 & 7.74 & -49.9461 \\
\hline 001 & 2.1942 & 2.2042 & 3.2519 & 3.2705 & 4.2648 & 4.2881 & 8.06 & -29.4682 & 7.74 & -49.9500 \\
\hline 200 & 2.2199 & 2.2300 & 3.2161 & 3.2345 & 4.2409 & 4.2640 & 8.06 & -29.4673 & 7.75 & -49.9450 \\
\hline 102 & 2.2099 & 2.2200 & 3.3210 & 3.3400 & 4.3412 & 4.3649 & 8.06 & -29.4811 & 7.79 & -51.1006 \\
\hline 003 & 2.2090 & 2.2191 & 3.3889 & 3.4082 & 4.4084 & 4.4324 & 8.04 & -30.4813 & 7.81 & -51.6613 \\
\hline 600 & 2.2781 & 2.2885 & 3.2562 & 3.2748 & 4.3079 & 4.3313 & 8.06 & -29.5662 & 7.79 & -49.9224 \\
\hline 203 & 2.2253 & 2.2355 & 3.3962 & 3.4156 & 4.4235 & 4.4476 & 8.03 & -31.0126 & 7.82 & -51.6176 \\
\hline 105 & 2.2053 & 2.2154 & 3.4967 & 3.5167 & 4.5148 & 4.5394 & 7.86 & -52.2952 & 7.86 & -52.3249 \\
\hline
\end{tabular}

${ }^{a}$ All distances $r, R$, and $R_{m}$ (giving the position of energy minimum) are in Bohr $\left(a_{0}\right) .{ }^{b} \epsilon$ is the well depth in $\mathrm{cm}^{-1}$. ${ }^{c}$ See the text for a definition of scaled distances.
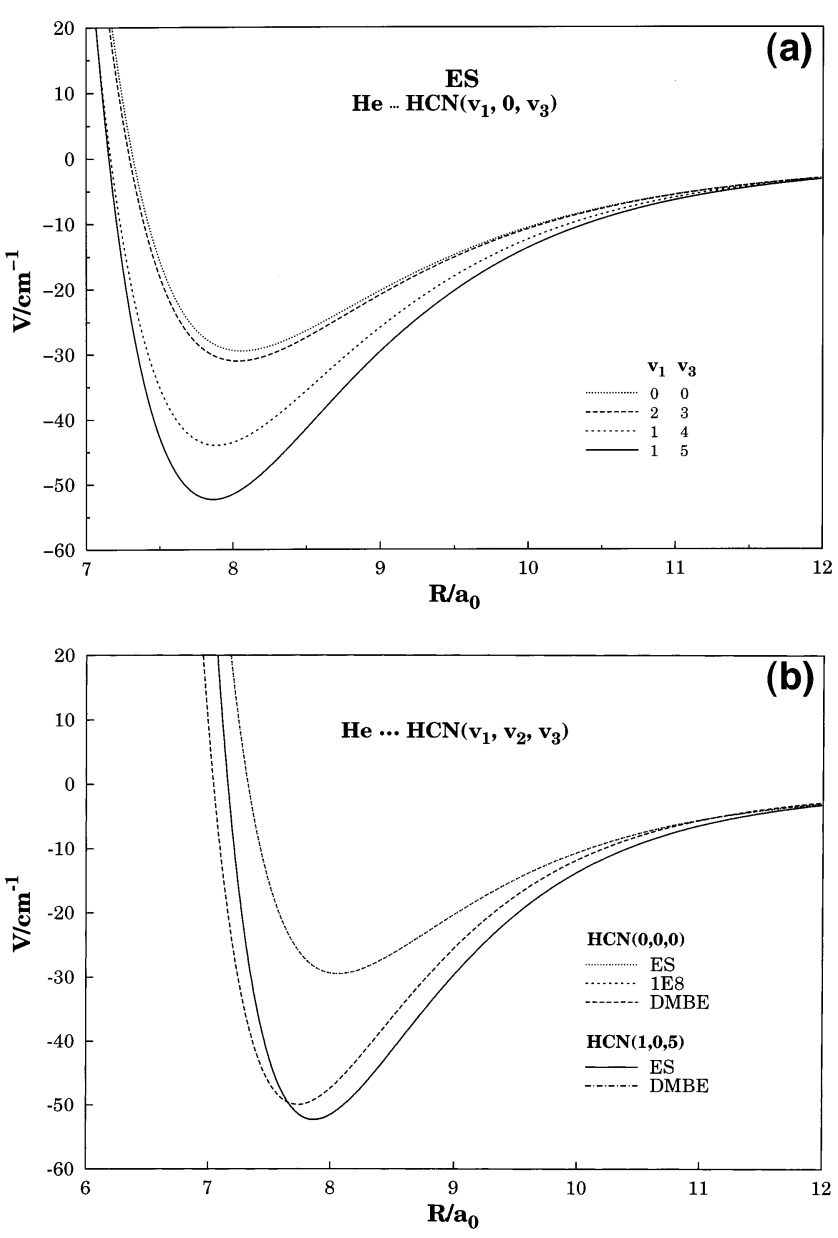

Figure 4. Comparison of potential wells of different states of $\mathrm{HeHCN}$. Panel (a): (i) $(1,0,5) / \mathrm{ES}$, (ii) $(1,0,5) / \mathrm{DMBE}$, (iii) $(0,0,0) / \mathrm{ES}$, (iv) $(0,0,0) / 1 \mathrm{E} 8$, and (v) $(0,0,0) / \mathrm{DMBE}$. Panel (b): (i) $(0,0,0) / 1 \mathrm{E} 8$, (ii) $(2,0,3) / \mathrm{ES}$, (iii) $(1,0,4) / \mathrm{ES}$, and (iv) $(1,0,5) / \mathrm{ES}$.

value zero to make the molecule remain linear). In both cases, we obtain identical results as discussed below.

The well is usually deeper, though to a small extent, in higher states corresponding to greater stretch, as may be seen in Figure 4. For a particular stretch, the repulsive DMBE wall completely coincides with the $1 \mathrm{E} 8$ ab initio surface in the repulsive region, [see Figure 1c]. Thus, Figure 4a compares representative ES wells for different stretches in states $(2,0,3),(1,0,4)$, and $(1,0,5)$ of HCN against that of the 1E8 $(0,0,0)$ state [ES and 1E8 wells coincide for the $(0,0,0)$ state)], whereas Figure $4 \mathrm{~b}$ compares DMBE, ES, and 1E8 wells for the HCN vibrational state $(0,0,0)$ on one hand and compares DMBE wells for the $(0,0,0)$ and $(1,0,5)$ states on the other [the ES and DMBE wells of state
$(1,0,5)$ coincide]. The ES well for the most stretched state $(1,0,5)$ is seen to be the deepest solely on account of the larger contribution of DMBE to the ES formulation. The wells obtained by the DMBE and ES methods for representative states have been collected in Table 2, along with effective linear $\mathrm{H}-\mathrm{C}-\mathrm{N}$ distance obtained as sum of expectation values of $\mathrm{CN}$ and $\mathrm{CH}$ bond lengths scaled by a factor so as to have these bond lengths in the $(0,0,0)$ state equal to their equilibrium values. For example, the expectation values of $r(\mathrm{CN})$ and $r(\mathrm{OH})$, where $\mathrm{O}$ is center of mass of $\mathrm{CN}$, are $2.2020 a_{0}$ and $3.2143 a_{0}$ in state $(0,0,0)$. They were multiplied by scaling factors 0.99546 and 0.99433 to obtain their equilibrium values 2.1920 and 3.1961 $a_{0}$, respectively. The same scaling factors were used to scale $r(\mathrm{CN})$ and $r(\mathrm{OH})$ bond lengths/distances of all other states. Both the DMBE and the ES wells obtained with inward or outward stretch may be seen to go deeper similarly. We have studied bound states with $\mathrm{CN}$ and $\mathrm{CH}$ bonds stretched according to different vibrational modes. Table 3 presents values calculated for some of these stretches of the two frequencies for groundstate transitions $J=1 \leftarrow 0$ and $J=2 \leftarrow 1$ that were observed at 15.9 and $31.3 \mathrm{GHz}$ by Drucker et al. ${ }^{4}$ They are discussed later.

3.2. Topographical Features. Though topographically the ES and 1E8 potential energy surfaces look similar [Figure 2, parts $\mathrm{c}$ and a], there is an important difference; the ES has the correct short and long-range behavior as it entails the salient features of both the DMBE and 1E8 surfaces. As shown in Figure 1a, the ES scheme warrants a smooth transition from the high energy regions to low energy regions, where the van der Waals potential $V_{2}$ (here, 1E8) is valid and offers (see below) a good representation of bound states. For high energy regions, the DMBE provides better promise for kinetics studies. ${ }^{7}$ The ES potential energy surface can therefore be expected to offer both the bound-state and dynamical properties of the system. In fact, our dynamics studies are in progress.

3.3. Bound States and Microwave Transitions. The bound state levels obtained from the ES energy surface are shown in Figure 5. The Figure also shows, for comparison, rovibrational levels as obtained from the 1E8 surface. Clearly, the two sets of levels are very close together and are indistinguishable within the scale of Figure 5. However, the ES levels are slightly lower compared to $1 \mathrm{E} 8$ levels in general. The frequencies of the corresponding microwave transitions are given in Table 4. They may be seen to be in good agreement with experiment. The $1 \mathrm{E} 8$ frequencies are also given in Table 4 for comparison.

The first two transitions observed at 15.9 and $31.3 \mathrm{GHz}$ by Drucker et al. $^{4}$ are well brought out by our bound-state calculations using the ES potential energy surface. The calculations also bring out successfully the transitions observed at 101.4, 105.8, and 4.6 GHz. However, the one observed by 
TABLE 3: Microwave Frequencies of States of Different Stretching Modes

\begin{tabular}{|c|c|c|c|c|c|}
\hline \multirow{3}{*}{$\begin{array}{c}\text { assignment } \\
v_{1}, v_{2}, v_{3} \text { parity }\end{array}$} & \multirow{3}{*}{$\begin{array}{c}\text { rotational constant }{ }^{a} \\
B_{e}, \mathrm{~cm}^{-1}\end{array}$} & \multicolumn{4}{|c|}{ frequency $/ \mathrm{MHz}$} \\
\hline & & \multicolumn{2}{|c|}{$v_{1}$ for $j, L, J(0,1,1) \leftarrow(0,0,0)$} & \multicolumn{2}{|c|}{$v_{2}$ for $j, L, J(0,2,2) \leftarrow(0,1,1)$} \\
\hline & & observed $^{b}$ & ES & observed & ES \\
\hline $000 \mathrm{e}$ & $1.478221834^{b}$ & 15893.6108 & 15893.6108 & 31325.2443 & 31325.3070 \\
\hline $001 \mathrm{e}$ & 1.46815 & & 15882.9424 & & 31299.3279 \\
\hline $100 \mathrm{e}$ & 1.46780 & & 15882.5751 & & 31298.4279 \\
\hline $200 \mathrm{e}$ & 1.45708 & & 15870.9654 & & 31270.1407 \\
\hline $102 \mathrm{e}$ & 1.44805 & & 15860.9619 & & 31245.7727 \\
\hline $003 \mathrm{e}$ & 1.44603 & & 15858.6883 & & 31240.2460 \\
\hline $203 \mathrm{e}$ & 1.42711 & & 15837.0345 & & 31187.4400 \\
\hline
\end{tabular}

${ }^{a}$ From ref $47 .{ }^{b}$ From ref 4.

TABLE 4: Comparison of ES and 1E8 Microwave Frequencies $(v)$ in $\mathrm{MHz}$

\begin{tabular}{lrrr}
\hline $\begin{array}{c}\text { assignments of } \\
\text { frequency }(j, L, J)\end{array}$ & $v^{a}$ (observed) & \multicolumn{1}{c}{ 1E8 } & \multicolumn{1}{c}{ ES } \\
\hline$(0,1,1) \leftarrow(0,0,0)$ & 15893.6108 & 15893.6125 & 15893.6108 \\
$(0,2,2) \leftarrow(0,1,1)$ & 31325.2443 & & 31325.3070 \\
$24 D_{j}$ & 461.9773 & 461.9825 & 461.9145 \\
$(1,1,2) \leftarrow(0,1,1)$ & 101432.0813 & 101432.1050 & 101421.9844 \\
$(1,2,3) \leftarrow(0,2,2)$ & 105795.3110 & 105795.0313 & 105782.1624 \\
$(1,2,3) \leftarrow(1,2,2)$ & 4604.2000 & 4604.4200 & 4598.5816 \\
$(1,0,1) \leftarrow(0,0,0)$ & & 98603.1200 & 98594.9390 \\
$(1,1,1) \leftarrow(0,1,1)$ & & 97892.1300 & 97885.3896 \\
$(0,3,3) \leftarrow(0,2,2)$ & & 45767.1200 & 45769.6546 \\
$(1,3,3) \leftarrow(0,3,3)$ & & 106633.3200 & 106626.1382
\end{tabular}

${ }^{a}$ From ref $4 .{ }^{b}$ From ref $5 .{ }^{c}$ This work.

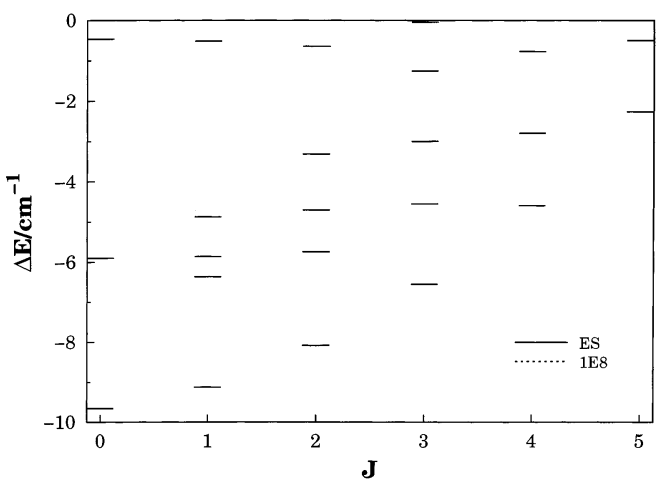

Figure 5. Comparison of rovibrational levels of $\mathrm{HeHCN}$ : (a) ES and (b) $1 \mathrm{E} 8$.

Drucker et al. ${ }^{4}$ at $101.2 \mathrm{GHz}$ could not be obtained both by our ES or the 1E8 calculations of Atkins and Hutson. ${ }^{5}$ However, this transition might be associated with some stretching mode. The transition $(j, L, J)=(1,1,2) \leftarrow(0,1,1)$ observed at 101.4 $\mathrm{GHz}$ may get perturbed under stretch, leading to lowering of its frequency to $101.2 \mathrm{GHz}$. Such a lowering has been observed in our calculations (see Table 3 ). The ES potential energy surface thus seems to hold some promise to explain rovibrational levels and associated microwave transitions.

Although the DMBE levels are lower, the values of the corresponding frequencies agree fairly closely with those of experiment, ES and 1E8. They are, however, lower by about 1 GHz. It turns out that DMBE is closer to the 1A8 type of potential form proposed by Atkins and Hutson, ${ }^{5}$ as the two predict such spectroscopic properties more similarly.

Coming to the next heavier member of $\mathrm{Rg}-\mathrm{HCN}$ series, the frequency of the lowest $J=1 \leftarrow 0$ rovibrational transition was calculated to be $5.52 \mathrm{~cm}^{-1}$ by the ES method ${ }^{8}$ for $\mathrm{Ar}-\mathrm{HCN}$. This may be compared to the corresponding frequency of $\mathrm{HeHCN}$ which is about $0.53 \mathrm{~cm}^{-1}$ or about $15893 \mathrm{MHz}$ (vide Table 4).
It may be noted that bound states of different rovibrational levels corresponding to different stretching of $\mathrm{CN}$ and $\mathrm{CH}$ bonds may form an interesting spectroscopic study of HeHCN. Such a study can further help in understanding if different rovibrational transitions can occur in the same vibrational state as observed experimentally by Drucker et al. ${ }^{4}$ Some initial results are given in Table 3. It may be seen that the frequency of transition generally decreases with greater stretch associated with higher energy states.

\section{Concluding Remarks}

The global 6D potential energy surfaces for HeHCN have been calculated by the DMBE and ES methods. They correctly behave at all dissociation limits. However, the ES surface reproduces the properties of the van der Waals molecule better. The quality of the surface has been examined by its ability to reproduce the frequencies of rovibrational transitions. Suitability of the surface to study varied spectroscopic features such as the effect of bond stretching on rovibrational levels and subsequent changes of frequencies of the corresponding microwave transitions has been investigated. The initial results as reported here are encouraging. Our study on dynamics of the reaction $\mathrm{He}+\mathrm{HCN} \rightarrow \mathrm{He}+\mathrm{H}+\mathrm{CN}$ is currently in progress. The ES potential energy surface reported in this paper is, to our knowledge, the only existing one which is capable of reproducing both the bound states and kinetics of the HeHCN molecule, although some important shortcoming may still be remaining.

Acknowledgment. W.H.A. thanks Dr. S. P. J. Rodrigues and P.J.B.S. Caridade, Departamento de Química, Universidade de Coimbra, Portugal, for computational assistance, and expresses his indebtedness to Professor N. Sathyamurthy of the Indian Institute of Technology, Kanpur, India, for continuous encouragements. This work has the support of Fundação para a Ciência e Tecnologia, Portugal.

\section{References and Notes}

(1) Green, S.; Thaddeus, P. Astrophys. J. 1974, 104, 653 7666.

(2) Clary, D. C.; Dateo, C. E.; Stoecklin, T. J. Chem. Phys. 1990, 93 ,

(3) Gutowsky, H. S.; Keen, J. D.; Germann, T. C.; Emilsson, T. J. Chem. Phys. 1993, 98, 6801. 2646.

(4) Drucker, S.; Tao, F.-H.; Klemperer, W. J. Phys. Chem. 1995, 99,

(5) Atkins, K. M.; Hutson, J. M. J. Chem. Phys. 1996, 105, 440

(6) Umera, K.; Hara, A.; Tanaka, K. J. Chem. Phys. 1996, 104, 9747.

(7) Rodrigues, S. P. J.; Varandas, A. J. C. J. Phys. Chem. A 1998, 102,6266 .

(8) Varandas, A. J. C.; Rodrigues, S. P. J.; Gomes, P. A. J. Chem. Phys. Lett. 1998, 297, 458.

(9) Cybulski, S. M.; Couvillion, J.; Klos, J.; Chalasinski, G. J. Chem. Phys. 1999, 110, 1416.

(10) Tanaka, K.; Bailleux, S.; Mizoguchi, A.; Harda, K.; Baba, T.; Ogawa, I.; Shirasaka, M. J. Chem. Phys. 2000, 113, 1524. 

4604.

(11) Toczylowski, R. R.; Cybulski, S. M. J. Chem. Phys. 2000, 112,

(12) Lee, H.-S.; McCoy, A. B.; Toczylowski, R. R.; Cybulski, S. M. J. Chem. Phys. 2000, 113, 5736.

(13) Cybulski, S. M.; Toczylowski, R. R.; Lee, H.-S.; McCoy, A. B. J. Chem. Phys. 2000, 113, 9549.

(14) Murdachaew, G.; Misquitta, A. J.; Bukowski, R.; Szalewicz, K. J. Chem. Phys. 2001, 114, 764 .

(15) Toczylowski, R. R.; Doloresco, F.; Cybulski, S. M. J. Chem. Phys. $\mathbf{2 0 0 1}, 114,851$

(16) Cambell, E. J.; Buxton, L.; Legon, A. C. J. Chem. Phys. 1983, 78 , 3483.

(17) Leopold, K. R.; Fraser, G. T.; Lin, F. J.; Nelson, D. D., Jr.; Klemperer, W. J. Chem. Phys. 1984, 81, 4922.

(18) Klots, T. D.; Dykstra, C. E.; Gutowsky, H. S. J. Chem. Phys. 1989 90,30

(19) Mladenović, M.; Bacic, Z. J. Chem. Phys. 1991, 94, 4988.

(20) Yaron, D.; Klemperer, W. J. Chem. Phys. 1991, 95, 1907.

(21) Cooksy, A. L.; Drucker, S.; Faeder, J.; Gottlieb, C. A.; Klemperer, W. J. Chem. Phys. 1991, 95, 3017.

(22) Tao, F.; Drucker, S.; Klemperer, W. J. Chem. Phys. 1995, 102, 7289.

(23) Bowman, J. M.; Padmavathi, D. A. Mol. Phys. 1996, 88, 21.

(24) Bumgarner, R. E.; Blake, G. A. Chem. Phys. Lett. 1989, 161, 308

(25) Fraser, G. T.; Pine, A. S. J. Chem. Phys. 1989, 91, 3319

(26) Varandas, A. J. C. Adv. Chem. Phys. 1988, 74, 255.

(27) Varandas, A. J. C. Chem. Phys. Lett. 1992, 194, 333.

(28) Varandas, A. J. C. In Dynamical Processes in Molecular Physics; Delgado-Barrio, G., Ed.; IOP Publishing: Bristol, 1993; p 3.

(29) Bruehl, M.; Schatz, G. C. J. Phys. Chem. 1988, 92, 7223.

(30) Hippler, H.; Schranz, H. W.; Troe, J. J. Phys. Chem. 1986, 90, 6158.

(31) Varandas, A. J. C.; Silva, J. D. J. Chem. Soc., Faraday Trans. 2 1986, 82,593 .
(32) Varandas, A. J. C.; Silva, J. D. J. Chem. Soc., Faraday Trans. 1992, 88,941

(33) Murrell, J. N.; Carter, S.; Farantos, S. C.; Huxley, P.; Varandas, A. J. C. Molecular Potential Energy Functions; Wiley: Chichester, U.K., 1984.

(34) Varandas, A. J. C. In Reaction and Molecular Dynamics; Laganá, A., Riganelli, A., Eds.; Springer: Berlin, 2000; Vol. 75 of Lecture Notes in Chemistry, p 33.

(35) Varandas, A. J. C.; Rodrigues, S. P. J. J. Chem. Phys. 1997, 106 9647.

(36) Varandas, A. J. C. Mol. Phys. 1987, 60, 527.

(37) Le Roy, R. J. Spec. Period. Rep. Chem. Soc. Mol. Spectrosc. 1973, $1,113$.

(38) Varandas, A. J. C. J. Chem. Phys. 1996, 105, 3524

(39) Varandas, A. J. C. J. Chem. Phys. 1997, 107, 867.

(40) Hutson, J. M. Comput. Phys. Comm. 1994, 84, 1.

(41) Hutson, J. M. BOUND computer code, version 5; distributed by Collaborative Computational Project No. 6 of the Science and Engineering Research Council, U.K., 1993.

(42) Dunning, T. H., Jr. J. Chem. Phys. 1989, 90, 1007.

(43) Kendall, R. A.; Dunning, T. H., Jr.; Harrison, R. J. J. Chem. Phys. 1992, 96, 6796.

(44) Woon, D. E.; Dunning, T. H., Jr. J. Chem. Phys. 1994, 100, 2975.

(45) Wilson, A. A.; Woon, D. E.; Peterson, K. A.; Dunning, T. H., Jr. J. Chem. Phys. 1999, 110, 7667.

(46) Werner, H.-J.; Knowles, P. J. MOLPRO is a package of ab initio programs written by H.-J. Werner, P. J. Knowles, with contributions from J. Almlöf, R. D. Amos, M. J. O. Deegan, S. T. Elbert, C. Hampel, W. Meyer, K. A. Peterson, R. Pitzer, A. J. Stone, P. R. Taylor, R. Lindh, 1998.

(47) Carter, S.; Mills, I. M.; Handy, N. C. J. Chem. Phys. 1993, 99 